\title{
AGGRESSIVE LONGITUDINAL AIRCRAFT PATH TRACKING USING NONLINEAR CONTROL
}

\author{
Saif A. Al-Hiddabi and N. Harris McClamroch*
}

\begin{abstract}
We study the problem of converting a trajectory tracking controller to a path tracking controller for a nonlinear non-minimum phase longitudinal aircraft model. The solution of the trajectory tracking problem is based on the requirement that the aircraft follows a given time parameterized trajectory in inertial frame. In this paper we introduce an alternative nonlinear control design approach called path tracking control. The path tracking approach is based on designing a nonlinear state feedback controller that maintains a desired speed along a desired path with closed loop stability. This design approach is different from the trajectory tracking approach where aircraft speed and position are regulated along the desired path. The path tracking controller regulates the position errors transverse to the desired path but it does not regulate the position error along the desired path. First, a trajectory tracking controller, consisting of feedforward and static state feedback, is designed to guarantee uniform asymptotic trajectory tracking. The feedforward is determined by solving a stable noncausal inversion problem. Constant feedback gains are determined based on LQR with singular perturbation approach. A path tracking controller is then obtained from the trajectory tracking controller by introducing a suitable state projection.
\end{abstract}

KeyWords: Longitudinal flight, path tracking, nonlinear control.

\section{INTRODUCTION}

In a trajectory tracking problem, the desired outputs, parameterized by time, are provided by a command generator. The trajectory tracking controller processes the desired outputs and forces the system outputs to follow the desired outputs as closely as possible. In the presence of tracking errors, the trajectory tracking controller attempts to make the outputs "catch up" with the time-parameterized desired outputs; this may lead to closed loop performance difficulties and to large control signals.

One approach to eliminate such problems is to use a path tracking controller instead of a trajectory tracking

Manuscript received June 1, 2000; revised October 2, 2000; accepted Junuary 11, 2001.

Saif A. Al-Hiddabi is with Department of Mechanical and Industrial Engineering, Sulatn Qaboos University, P.O. Box 33 Muscat-Al-Khod 123, Sultanate of Oman.

N. Harris McClamroch is with Department of Aerospace Engineering, The University of Michigan, Ann Arbor, MI 481092140, USA.

*Support from the National Science Foundation, Grant ECS9906018 , is gratefully acknowledged. controller. The objective of path tracking is to track a specified geometric path in the output space with specified "velocity" along the path. The path tracking controller eliminates the aggressiveness of the trajectory tracking controller by forcing convergence to the desired path in a smooth way.

Path tracking, maneuver regulation or path following controllers have been studied for robotic systems [7, $15]$ and for aerospace vehicles [10,12]. A general approach has been developed by Hauser and Hindman $[9,11]$ for feedback linearizable nonlinear control system.

The present paper introduces an alternative nonlinear control design approach for the nonlinear non-minimum phase longitudinal aircraft model, treated in [4], called path tracking. The path tracking controller regulates the position errors transverse to the desired path but it does not regulate the position error along the desired path. Based on our experience with the planar vertical take off and landing (PVTOL) aircraft model treated in [5] and the simplified longitudinal aircraft model treated in [3], this method improves closed loop properties and reduces the size of control inputs. The path tracking approach is based on designing a tracking controller that maintain a desired speed along a desired path with closed loop stability. 
This design approach is different from the trajectory tracking approach treated in [4] where aircraft speed and position are regulated along the desired path. This work represent an extension to the work of Hauser and Hindman modified for nonlinear non-minimum phase flight control problems.

As suggested in [1,6], first a trajectory tracking controller, consisting of feedforward and static state feedback, is designed to guarantee uniform asymptotic trajectory tracking. The feedforward is determined by solving a stable noncausal inversion problem. Constant feedback gains are determined based on LQR optimization and assumed satisfaction of a robustness inequality. A path tracking controller is obtained from the tracking controller by introducing a suitable state projection that is related to the LQR feedback gains. Properties of the closed loop, including local asymptotic convergence of the transverse errors, are developed.

\section{EQUATIONS OF MOTION OF THE FLIGHT VEHICLE}

We consider the longitudinal flight control model introduced in $[1,4]$ which describes the dynamics of an aircraft in forward flight. The longitudinal aircraft model provides a challenging example for nonlinear flight control studies. The aircraft model includes aerodynamic forces as well as coupling between the aerodynamic pitch moment and the aerodynamic translational forces. Figure 1 shows a prototype longitudinal aircraft in flight. The aircraft state is the position, $X, Z$, of the aircraft center of mass, the pitch angle $\theta$, of the aircraft, and the corresponding velocities, $\dot{X}, \dot{Z}, \dot{\theta}$. The control inputs, $T$ and $\mathbb{\mathbb { S }}$ are, respectively, the thrust along the aircraft body fixed $x$-axis and the pitching moment about the aircraft center of mass.

Deflecting an elevator upward produces a small negative lift force which generate a positive pitching moment about the center of mass of the aircraft. The presence of this parasitic aerodynamic force makes the longitudinal aircraft model non-minimum phase. In this case nonlinear control design method such as dynamic inversion is not directly applicable to this flight control problem [14].

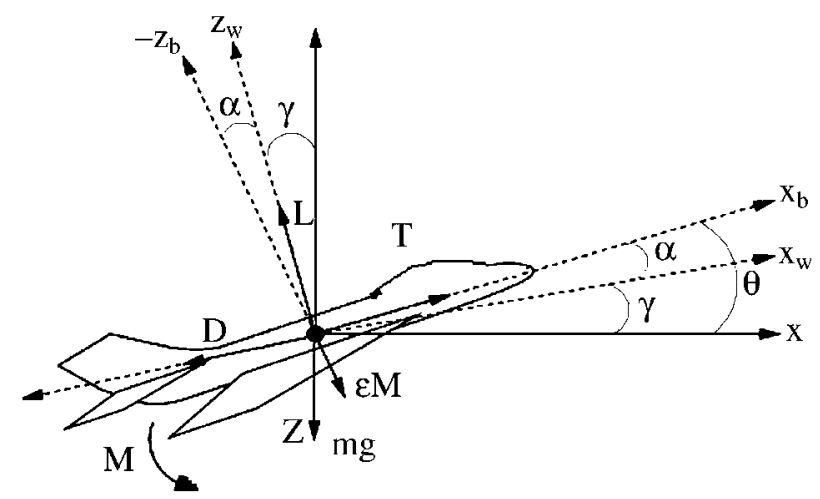

Fig. 1. Longitudinal aircraft model in flight.
The full longitudinal equations of motion of an aircraft can be written as:

$$
\begin{aligned}
& m \ddot{X}=-D \cos \gamma-L \sin \gamma+T \cos \theta \\
& -m \ddot{Z}=D \sin \gamma-L \cos \gamma-T \sin \theta+m g \\
& I_{y} \ddot{\theta}=\mathbb{Q} \mathbb{M} .
\end{aligned}
$$

The aerodynamic lift force $(L)$, the aerodynamic


are given by:

$$
\begin{aligned}
& L=\frac{1}{2} \rho V^{2} S C_{l}, \\
& D=\frac{1}{2} \rho V^{2} S C_{d}, \\
& \mathbb{\mathbb { N }}=\frac{1}{2} \rho V^{2} S \bar{c} C_{m},
\end{aligned}
$$

where $\rho$ is the air density, $V$ is the aircraft speed, $S$ is the reference wing area, $\bar{c}$ is the mean aerodynamic chord, $C_{l}$ is the lift coefficient, $C_{d}$ is the drag coefficient, and $C_{m}$ is the pitching moment coefficient. Note that the coefficient of lift $C_{l}$, the coefficient of $\operatorname{drag} C_{d}$, and the coefficient of pitching moment $C_{m}$ can be written as

$$
\begin{aligned}
& C_{l}=C_{l 0}+C_{l \alpha} \alpha+C_{l \delta_{e}} \delta_{e} \\
& C_{d}=C_{d 0}+\mathcal{K} C_{l}^{2} \\
& C_{m}=C_{m 0}+C_{m \alpha} \alpha+\frac{\bar{c}}{2 V}\left(C_{m q}+C_{m \dot{\alpha}}\right) \dot{\theta}-\frac{\bar{c} C_{m \dot{\alpha}}}{2 V} \dot{\gamma}+C_{m \delta_{e}} \delta_{e}
\end{aligned}
$$

where, $V_{2}=\dot{X}^{2}+\dot{Z}^{2}, \alpha=\theta-\tan ^{-1} \frac{\dot{Z}}{\dot{X}}$ is the angle of attack, $\delta_{e}$ is the elevator deflection and $C_{l 0}, C_{l \alpha}, C_{l \delta_{e}}, C_{d 0}, \mathcal{K}, C_{m 0}$, $C_{m \alpha}, C_{m q}, C_{m \dot{\alpha}}, C_{m \delta_{e}}$ are assumed constants. The rate of change of flight path angle $\dot{\gamma}$ in equation (9) is

$$
\dot{\gamma}=\frac{m(\dot{X} \ddot{Z}-\dot{Z} \ddot{X})}{m V^{2}}
$$

Define $Q_{s}=\frac{1}{2} \rho V^{2} S, \tilde{C}_{l}=C_{l 0}+C_{l \alpha} \alpha$ and $\tilde{C}_{d}=C_{d 0}+$ $\mathcal{K} \widetilde{C}_{l}^{2}$. Then after substituting equations (1),(2) into equation (10), the aerodynamic lift force $(L)$, the aerodynamic drag force $(D)$, and the aerodynamic pitching moment (极) can be written as:

$$
\begin{aligned}
& L=Q_{s}\left[\tilde{C}_{l}+C_{l \delta_{e}} \delta_{e}\right] \\
& D=Q_{s}\left[\tilde{C}_{d}+2 \mathcal{K} C_{l \delta_{e}} \tilde{C}_{l} \delta_{e}+\mathcal{K} C_{l \delta_{e}}^{2} \delta_{e}^{2}\right]
\end{aligned}
$$




$$
\begin{aligned}
& \mathbb{\mathbb { Q } \Omega}=Q_{s} \bar{c}\left[C_{m 0}+C_{m \alpha} \alpha-\frac{\bar{c} C_{m \dot{\alpha}} \rho S}{4 m} \tilde{C}_{l}+\frac{\bar{c}}{2 V}\left(C_{m q}+C_{m \dot{\alpha}}\right) \dot{\theta}\right. \\
& +\frac{\bar{c} C_{m \dot{\alpha}} g}{2 V^{2}} \cos \gamma+\frac{\bar{c} C_{m \dot{\alpha}}}{2 m V^{2}} T \sin \alpha \\
& \left.+\left(C_{m \delta_{e}}-\frac{\bar{c} C_{m \dot{\alpha}} C_{l \delta_{e}} \rho S}{4 m}\right) \delta_{e}\right] .
\end{aligned}
$$

From equation (13), we can solve for $\delta_{e}$ and then substitute the result into equations $(11,12)$ to obtain:

$$
\begin{aligned}
L= & Q_{s}\left[\tilde{C}_{l 0}+\tilde{C}_{l \alpha} \alpha\right]-m g K_{1} \cos \gamma-K_{1} T \sin \alpha \\
& -K_{2} V \dot{\theta}+\epsilon_{0} \mathbb{\mathbb { I } \Omega} \\
D= & Q_{s}\left[C_{d 0}+\frac{\mathcal{K}}{Q_{s}^{2}} L^{2}\right],
\end{aligned}
$$

where

$$
\begin{aligned}
& \epsilon_{0}=\frac{C_{l \delta_{e}}}{\bar{c}\left[C_{m \delta_{e}}-\frac{\bar{c} C_{l \delta_{e}} C_{m \alpha} \rho S}{4 m}\right]}, K_{1}=\frac{\epsilon_{0} \bar{c}^{2} C_{m \alpha} \rho S}{4 m}, \\
& K_{2}=\frac{\epsilon_{0} \bar{c}^{2} \rho S\left(C_{m q}+C_{m \alpha}\right)}{4} \\
& \tilde{C}_{l 0}=\left(1+K_{1}\right) C_{l 0}+\epsilon_{0} \bar{c} C_{m 0}
\end{aligned}
$$

and

$$
\tilde{C}_{l \alpha}=\left(1+K_{1}\right) C_{l \alpha}+\epsilon_{0} \bar{c} C_{m \alpha}
$$

Equations (14), (15) show the relation between the aerodynamic lift $L$ and $\operatorname{drag} D$ and the aerodynamic pitching moment (还). The parameter $\epsilon_{0}$ in equation (14) gives the explicit coupling between the aerodynamic forces and the aerodynamic control moment. This parameter represents approximately the ratio between the aerodynamic lift force and the aerodynamic moment generated by the elevator. We scale the longitudinal aircraft model by dividing equations (1), (2) by $m g$, and dividing equation (3) by $I_{y}$. Define $x=X / g, z=-Z / g, u_{x}=T /(m g), u_{m}=\mathbb{T} / / I_{y}$ and $\epsilon=\epsilon_{0} I_{y} /(m g)$. Then the rescaled dynamics become

$$
\begin{aligned}
& \ddot{x}=-D^{\prime} \cos \gamma-L^{\prime} \sin \gamma+u_{x} \cos \theta \\
& \ddot{z}=-D^{\prime} \sin \gamma+L^{\prime} \cos \gamma+u_{x} \sin \theta-1 \\
& \ddot{\theta}=u_{m},
\end{aligned}
$$

where $L^{\prime}$ and $D^{\prime}$ are dimensionless lift and drag forces given by:

$$
\begin{aligned}
& L^{\prime}=a_{l} v^{2}(1+c \alpha)-K_{1} \cos \gamma-\frac{K_{2}}{m} v \dot{\theta}+\epsilon u_{m}-K_{1} u_{x} \sin \alpha \\
& D^{\prime}=a_{d} v^{2}\left(1+b(1+c \alpha)^{2}\right)+\frac{2 K_{3}}{v^{2}}\left[a_{l} v^{2}(1+c \alpha)-K_{1} \cos \gamma\right. \\
&\left.\quad-\frac{K_{2}}{m} v \dot{\theta}\right]\left[\epsilon u_{m}-K_{1} u_{x} \sin \alpha\right] \\
&+\frac{K_{3}}{v^{2}}\left[\left(\epsilon u_{m}-K_{1} u_{x} \sin \alpha\right)^{2}+\left(K_{1} \cos \gamma+\frac{K_{2}}{m} v \dot{\theta}\right)^{2}\right. \\
&\left.\quad-2 a_{l} v^{2}(1+c \alpha)\left(K_{1} \cos \gamma+\frac{K_{2}}{m} v \dot{\theta}\right)\right]
\end{aligned}
$$

where

$$
\begin{aligned}
& v=\frac{V}{g}, a_{l}=\frac{\rho g S \tilde{C}_{l 0}}{2 m}, a_{d}=\frac{\rho g S C_{d 0}}{2 m}, c=\frac{\tilde{C}_{l \alpha}}{\widetilde{C}_{l 0}}, \\
& b=\frac{K \widetilde{C}_{l 0}^{2}}{C_{d 0}}, K_{3}=\frac{2 m \mathcal{K}}{\rho g S} .
\end{aligned}
$$

The zero dynamics of the longitudinal aircraft system can be obtained by assuming that the aircraft is flying with a constant horizontal speed $\dot{x}_{c}^{*}$ at a constant altitude. In this case $\ddot{x}=\ddot{z}=\dot{z}=\gamma=0$ and $\alpha=\theta$. The analysis of the longitudinal aircraft zero dynamics can be found in $[1,4]$. It was shown that the zero dynamics of the above longitudinal aircraft model is unstable or the longitudinal aircraft system is non-minimum phase.

\section{THE TRACKING PROBLEM FORMULATION}

We consider the flight control problem studied in [6] where the aircraft is required to perform a nap of the earth (NOE) maneuver in a vertical plane. The NOE maneuver commands correspond to a fixed horizontal velocity command of $130 \mathrm{~m} / \mathrm{s}$ and to a vertical position command given by $z_{c}=(250 / 2) *(1-\cos (\pi * t / 60))(m)$.

The controller design in this case is complicated due to the fact that the longitudinal aircraft model is nonminimum phase and also not linear affine in the control. In $[1,4]$, a two degrees of freedom trajectory tracking controller was designed for the nonlinear non-minimum phase longitudinal aircraft model described by equations (16), (17), (18) using the control architecture shown in Fig. 2.

The resulting trajectory tracking controller from [1, 4] can be summarized as:

(1) A state feedback controller that partially linearize the longitudinal aircraft model (16), (17), (18) is designed as

$$
\begin{gathered}
\epsilon u_{m}=\left(1+K_{1}\right) u_{x} \sin \alpha+\left(v_{z}+K_{1}+1\right) \cos \gamma \\
-v_{x} \sin \gamma-a_{l} v^{2}(1+c \alpha) .
\end{gathered}
$$




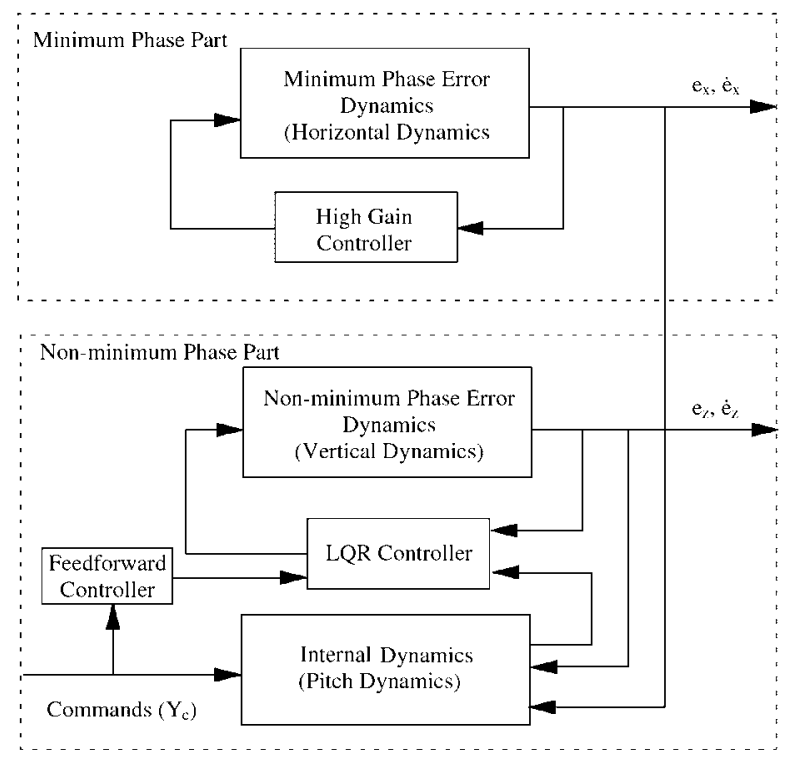

Fig. 2. Block diagram of the trajectory tracking closed loop.

where the thrust control $u_{x} \geq 0$ satisfies the quadratic equation

$\delta(\bar{x}) u_{x}^{2}+b(\bar{x}, \bar{v}) u_{x}+c(\bar{x}, \bar{v})=0$.

Here $\bar{v}=\left(v_{x}, v_{z}\right)$ is a new control variables, $\bar{x}=(x, \dot{x}$, $z, \dot{z}, \theta, \dot{\theta})$, and

$\delta(\bar{x})=K_{3}\left(\frac{\sin \alpha}{v}\right)^{2} \cos \alpha$,

$b(\bar{x}, \bar{v})=\frac{K_{3}}{v^{2}} \sin 2 \alpha\left[\left(v_{z}+1\right) \cos \gamma-v_{x} \sin \gamma\right]-\cos ^{2} \alpha$,

$c(\bar{x}, \bar{v})=\frac{K_{3}}{v^{2}}\left[\left(v_{z}+1\right) \cos \gamma-v_{x} \sin \gamma\right]^{2} \cos \alpha$

$+\left[\left(v_{z}+1\right) \cos \gamma-v_{x} \sin \gamma\right] \sin \alpha$

$+\left(v_{z}+1\right) \sin \theta+v_{x} \cos \theta+a_{d} v^{2} \cos \alpha$.

Note that $\delta(\bar{x})$ in equation (22) is small (dividing by aircraft speed), and in this case the solution of equation (22) can be written in a perturbation form as

$u_{x}=-\frac{c(\bar{x}, \bar{v})}{b(\bar{x}, \bar{v})}+\Delta u_{x}$

where $\Delta u_{x}$ is the change in $u_{x}$. Substitute this into equation (22) and solve for $\Delta u_{x}$ (ignoring products of small terms). An approximate solution of equation (22) can be then written as $u_{x}=-c(\bar{x}, \bar{v}) \frac{[b(\bar{x}, \bar{v})]^{2}+\delta(\bar{x}) c(\bar{x}, \bar{v})}{[b(\bar{x}, \bar{v})]^{3}}$.

Using the new control variables $\left(v_{x}, v_{z}\right)$, the longitudinal aircraft model described by equations (16), (17), (18) can be written in the normal form as

$$
\begin{aligned}
\ddot{x}= & v_{x} \\
\ddot{z}=v_{z} & \\
\epsilon \ddot{\theta}= & \left(1+K_{1}\right) u_{x} \sin \alpha+\left(v_{z}+K_{1}+1\right) \cos \gamma \\
& \quad-v_{x} \sin \gamma-a_{l} v^{2}(1+c \alpha) .
\end{aligned}
$$

(2) A feedforward controller is then designed using the bounded solutions $\theta^{*}$ and $\dot{\theta}^{*}$ of the driven dynamics

$$
\epsilon \ddot{\theta}^{*}=\left(1+K_{1}\right) u_{x}^{*} \sin \alpha^{*}+\left(\ddot{z}_{c}+K_{1}+1\right) \cos \gamma
$$

$$
-a_{l}\left(\dot{x}_{c}^{2}+\dot{z}_{c}^{2}\right)\left(1+c \alpha^{*}\right) .
$$

Here $\gamma=\tan ^{-1}\left(\dot{z}_{c} / \dot{x}_{c}\right), \alpha^{*}=\theta^{*}-\alpha$ and $u_{x}^{*}$ satisfies

$$
\delta\left(\theta^{*}, t\right) u_{x}^{* 2}+b\left(\theta^{*}, t\right) u_{x}^{*}+c\left(\theta^{*}, t\right)=0
$$

where

$$
\begin{aligned}
\delta\left(\theta^{*}, t\right)= & K_{3}\left(\frac{\sin \alpha^{*}}{\sqrt{\dot{x}_{c}^{2}+\dot{z}_{c}^{2}}}\right)^{2} \cos \alpha^{*}, \\
b\left(\theta^{*}, t\right)= & \frac{K_{3}}{\dot{x}_{c}^{2}+\dot{z}_{c}^{2}} \sin 2 \alpha^{*}\left[\left(\ddot{z}_{c}+1\right) \cos \gamma\right]-\cos ^{2} \alpha^{*}, \\
c\left(\theta^{*}, t\right)= & \frac{K_{3}}{\dot{x}_{c}^{2}+\dot{z}_{c}^{2}}\left[\left(\ddot{z}_{c}+1\right) \cos \gamma\right]^{2} \cos \alpha^{*} \\
& +\left[\left(\ddot{z}_{c}+1\right) \cos \gamma\right] \sin \alpha^{*}+\left(\ddot{z}_{c}+1\right) \sin \theta^{*} \\
& +a_{d}\left(\dot{x}_{c}^{2}+\dot{z}_{c}^{2}\right) \cos \alpha^{*} .
\end{aligned}
$$

The bounded solutions are obtained numerically using the iterative method introduced in [8]. The results are shown in Fig. 3.

(3) Finally a static state feedback controller that guarantees uniform asymptotic trajectory tracking is designed based on the decomposition shown in Fig. 2 as

$$
\begin{aligned}
& v_{x}=\ddot{x}_{c}-\frac{\beta_{1}}{\epsilon_{1}}\left(\dot{x}-\dot{x}_{c}\right)-\frac{\beta_{2}}{\epsilon_{1}^{2}}\left(x-x_{c}\right) \\
& v_{z}=\ddot{z}_{c}-k_{1}\left(z-z_{c}\right)-k_{2}\left(\dot{z}-\dot{z}_{c}\right)-k_{3}\left(\theta-\theta^{*}\right)-k_{4}\left(\dot{\theta}-\dot{\theta}^{*}\right)
\end{aligned}
$$



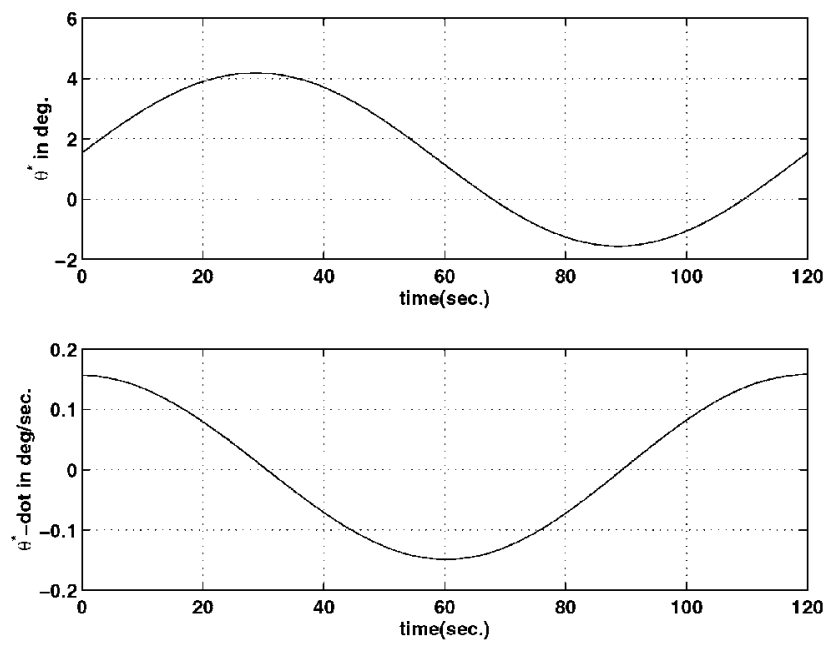

Fig. 3. Non-causal solution of the aircraft driven dynamics: NOE maneuver.

The feedback controller gains from [4] are given by $\epsilon_{1}=1, \beta_{1}=6, \beta_{2}=8,\left[k_{1}, k_{2}, k_{3}, k_{4}\right]=[-2.2361,-12$. $2245,-51.9616,-8.6262]$.

Note that this trajectory tracking controller requires non-causal knowledge of the complete tracking commands and their derivatives plus knowledge of the state for feedback.

To summarize the resulting trajectory tracking closed loop system, we introduce the following notations: Errors coordinate $e_{x}=x-x_{c}, e_{z}=z-z_{c}, \bar{\eta}=\eta-\eta_{s}$, where $\eta=(\theta$, $\dot{\theta})$ and $\eta_{s}=\left(\theta^{*}, \dot{\theta}^{*}\right)$. Define the commands vector as $Y_{c}=$ $\left(x_{c}, \dot{x}_{c}, z_{c}, \dot{z}_{c}\right)$ and the non-minimum phase states as $\bar{z}=\left(e_{n}\right.$, $\bar{\eta})$, where $e_{n}=\left(e_{z}, \dot{e}_{z}\right)$. Then the exact closed loop, in the error coordinates, using the above two degrees of freedom controller can be written in perturbation form $[1,4]$ as:

$$
\begin{aligned}
& \ddot{e}_{x}=-\frac{\beta_{1}}{\epsilon_{1}} \dot{e}_{x}-\frac{\beta_{2}}{\epsilon_{1}^{2}} e_{x}, \\
& \dot{\bar{z}}=A_{c} \bar{z}+\hat{g}\left(e_{x}, \dot{e}_{x}, e_{n}, \bar{\eta}+\eta_{s}, Y_{c}\right)-\hat{g}\left(0,0,0, \eta_{s}, Y_{c}\right) .
\end{aligned}
$$

Here the constant matrix $A_{c}$ is Hurwitz.

In [4] we have introduced robustness inequality for the above closed loop system that guarantees local uniform asymptotic trajectory tracking of the desired output commands with closed loop stability. Proofs of local uniform asymptotic trajectory tracking for such systems can be found in $[2,4,6,13]$. Thus according to $[9,11]$, the above trajectory tracking controller is suitable for conversion to a path tracking (or maneuver regulation) controller.

\section{THE PATH TRACKING PROBLEM}

In this section we design a path tracking controller for the aircraft model, so that its center of mass tracks a path in the vertical plane described by a fixed horizontal velocity of $\dot{x}_{c}=130 \mathrm{~m} / \mathrm{s}$ an altitude change given by the equation $z_{c}=(250 / 2) *(1-\cos (\pi * t / 60))(m)$. The path tracking controller regulates the position errors transverse to the desired path but it does not regulate the position error along the desired path. To formalize this approach, we introduce the following notation:

$$
\begin{aligned}
& \tilde{Y}_{p}=(x, \dot{x}), \tilde{Y}_{n}=(z, \dot{z}), \tilde{Y}=\left(\tilde{Y}_{p}, \tilde{Y}_{n}\right), \eta=(\theta, \dot{\theta}) \\
& \tilde{Y}_{p_{c}}=\left(x_{c}, \dot{x}_{c}\right), \tilde{Y}_{n_{c}}=\left(z_{c}, \dot{z}_{c}\right), \tilde{Y}_{c}=\left(\tilde{Y}_{p_{c}}, \tilde{Y}_{n_{c}}\right) \\
& \eta_{s}=\left(\theta^{*}, \dot{\theta}^{*}\right)
\end{aligned}
$$

Let $P_{11}$ be the positive definite solution of the Lyapunov equation

$$
P_{11} A_{11}+A_{11}^{T} P_{11}=-I_{2 \times 2} \text {, where } A_{11}=\left[\begin{array}{cc}
0 & 1 \\
-\beta_{2}-\beta_{1}
\end{array}\right] .
$$

Define the projection matrix $\tilde{P}_{6 \times 6}$ as

$$
\tilde{P}_{6 \times 6}=\left[\begin{array}{cc}
M\left(\epsilon_{1}\right) P_{11} M\left(\epsilon_{1}\right) & 0 \\
0 & P
\end{array}\right],
$$

where $M\left(\epsilon_{1}\right)=\operatorname{diag}\left(1 / \epsilon_{1}^{2}, 1 / \epsilon_{1}\right), \epsilon_{1}>0$ is the time scale parameter that was introduced in the trajectory tracking control and $P$ is the positive definite solution of algebraic Riccati equation

$$
A^{T} P+P A+Q-P B R^{-1} B^{T} P=0
$$

where $A$ is obtained from linearizing equations (24) and (25) under the assumption that the aircraft is flying horizontal at a fixed speed of $\dot{x}_{c}=130 \mathrm{~m} / \mathrm{s}, Q$ is positive definite matrix, $R>0, B=(0,1,0,1)$.

Following the same procedure as in $[1,6]$, we define path variable for each $(\tilde{Y}(t), \eta(t))$, as

$$
\pi(\tilde{Y}, \eta)=\underset{0 \leq \tau \leq 120 \text { sec. }}{\arg \min }\left\|\left[\begin{array}{c}
\tilde{Y}_{p}(t)-\tilde{Y}_{p_{c}}(\tau) \\
{\left[\tilde{Y}_{n}(t)-\tilde{Y}_{n_{c}}(\tau), \eta(t)-\eta_{s}(\tau)\right]^{T}}
\end{array}\right]\right\|_{\tilde{P}}^{T} .
$$

Note that $\|x\|_{\tilde{P}}:=\sqrt{x^{T} \tilde{P} x}, x \in R^{6}$.

Define transverse errors as $\bar{e}_{p}=\tilde{Y}_{p}-\tilde{Y}_{p_{c}}(\pi)$ and $\bar{z}=$ $\left(\bar{e}_{n}, \bar{\xi}\right)$, where $\bar{e}_{n}=\tilde{Y}_{n}-\tilde{Y}_{n_{c}}(\pi)$ and $\bar{\xi}=\eta-\eta_{s}(\pi)$. Following the approach of $[9,11]$, the maneuver regulation controller is thus obtained by replacing the explicit time variable in the trajectory tracking controller (28) and (29) 
by the projection variable $\pi$ as

$$
\begin{aligned}
& v_{x}= \ddot{x}_{c}(\pi)-\frac{\beta_{1}}{\epsilon_{1}}\left(\dot{x}-\dot{x}_{c}(\pi)\right)-\frac{\beta_{2}}{\epsilon_{1}^{2}}\left(x-x_{c}(\pi)\right) \\
& v_{z}=\ddot{z}_{c}(\pi)-k_{1}\left(z-z_{c}(\pi)\right)-k_{2}\left(\dot{z}-\dot{z}_{c}(\pi)\right) \\
&-k_{3}\left(\theta-\theta^{*}(\pi)\right)-k_{4}\left(\dot{\theta}-\dot{\theta}^{*}(\pi)\right)
\end{aligned}
$$

Note that the path tracking controller (34), (35) is in the form of nonlinear feedback and requires on-line solution of the minimization problem (33). We emphasize that the path tracking controller (34), (35) is not causal with respect to the command; the complete command is required to compute the bounded solution of the driven dynamics (26). This again reflects the non-minimum phase features of the longitudinal aircraft model.

Define $\hat{e}_{p}=M\left(\epsilon_{1}\right) \bar{e}_{p}$, then the path tracking closed loop in the transverse errors coordinate can be written in perturbation from as

$$
\begin{aligned}
& {\left[\begin{array}{c}
\epsilon_{1} \dot{\hat{e}}_{p} \\
\dot{\bar{z}}
\end{array}\right]=\left[\begin{array}{cc}
A_{11} & 0 \\
0 & A_{c}
\end{array}\right]\left[\begin{array}{c}
\hat{e}_{p} \\
\bar{z}
\end{array}\right]+\left[\begin{array}{c}
\epsilon_{1} M\left(\epsilon_{1}\right) \tilde{Y}_{p_{c}^{\prime}}(\pi) \\
\left.\left[\begin{array}{r}
\tilde{Y}_{n}^{\prime} \\
n_{c}
\end{array}\right], \eta_{s}^{\prime}(\pi)\right]^{T}
\end{array}\right](1-\dot{\pi})} \\
& +\left[\begin{array}{c}
0 \\
\hat{g}\left(\epsilon_{1} \hat{e}_{p}+\tilde{Y}_{p_{c}}(\pi), \bar{e}_{n}+\tilde{Y}_{n_{c}}(\pi), \bar{\xi}+\eta_{s}(\pi), \ddot{z}_{c}(\pi)\right)-\hat{g}\left(\tilde{Y}_{p_{c}}(\pi), \tilde{Y}_{n_{c}}(\pi), \eta_{s}(\pi), \ddot{z}_{c}(\pi)\right)
\end{array}\right]
\end{aligned}
$$

where $A_{11}$ and $A_{c}$ are Hurwitz.

In $[1,6]$ we have introduced robustness inequality for the above path tracking closed loop system that guarantees the following local results:

(a) if $(\tilde{Y}(0), \eta(0))=\left(\tilde{Y}_{c}(\bar{\pi}), \eta_{s}(\bar{\pi})\right)$ for some $\bar{\pi} \in R$, the output response satisfies

$$
\tilde{Y}(t)=\tilde{Y}_{c}(t+\bar{\pi}), \eta(t)=\eta_{s}(t+\bar{\pi}), t \geq 0
$$

and hence $x(t)=x_{c}(t+\bar{\pi})$ and $z(t)=z_{c}(t+\bar{\pi}), t \geq 0.6$;

(b) there is $r>0$ such that for all initial conditions satisfying

$$
\min _{\tau \in R}\left\|(\tilde{Y}(0), \eta(0))-\left(\tilde{Y}_{c}(\tau), \eta_{s}(\tau)\right)\right\|<r,
$$

the output response satisfies

$\tilde{Y}(t)-\tilde{Y}_{c}(\pi) \rightarrow 0, \eta(t)-\eta_{s}(\pi) \rightarrow 0$, as $t \rightarrow \infty$,

and hence $x(t)-x_{c}(\pi) \rightarrow 0$ and $z(t)-z_{c}(\pi) \rightarrow 0$ as $t \rightarrow \infty$.
Proofs of these results follow from [1].

The result (a) simply states that if the aircraft is initialized on the desired path $\left(x_{c}, \dot{x}_{c}, z_{c}, \dot{z}_{c}, \theta^{*}, \dot{\theta}^{*}\right)$ with zero transverse errors then the aircraft will continue flying the desired path with the desired speed without correcting for along path errors (note that the along path errors may not be initially zero in this case). The result (b) states that if the aircraft is initialized on a tube of radius $r$ from the desired path $\left(x_{c}, \dot{x}_{c}, z_{c}, \dot{z}_{c}, \theta^{*}, \dot{\theta}^{*}\right)$ then the aircraft will converge asymptotically to the desired path by correcting only for the transverse errors and not the along path errors.

\section{Simulations and comparison}

Simulations in Fig. 4 to Fig. 7, are obtained assuming that the aircraft initial condition is $\bar{x}(0)=(2000,130,34$.

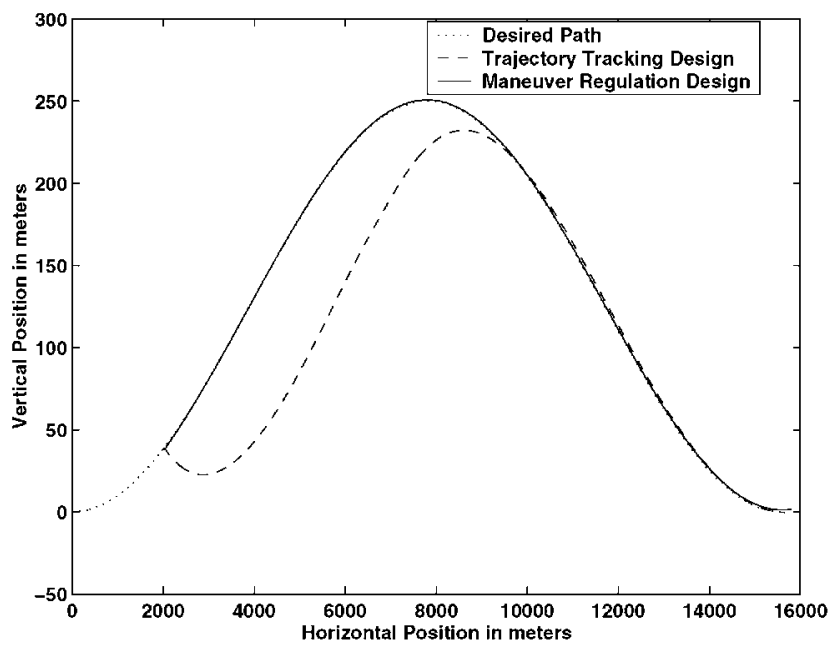

Fig. 4. Path tracking responses and trajectory tracking responses for NOE maneuver for the longitudinal aircraft model, non-zero initial errors.
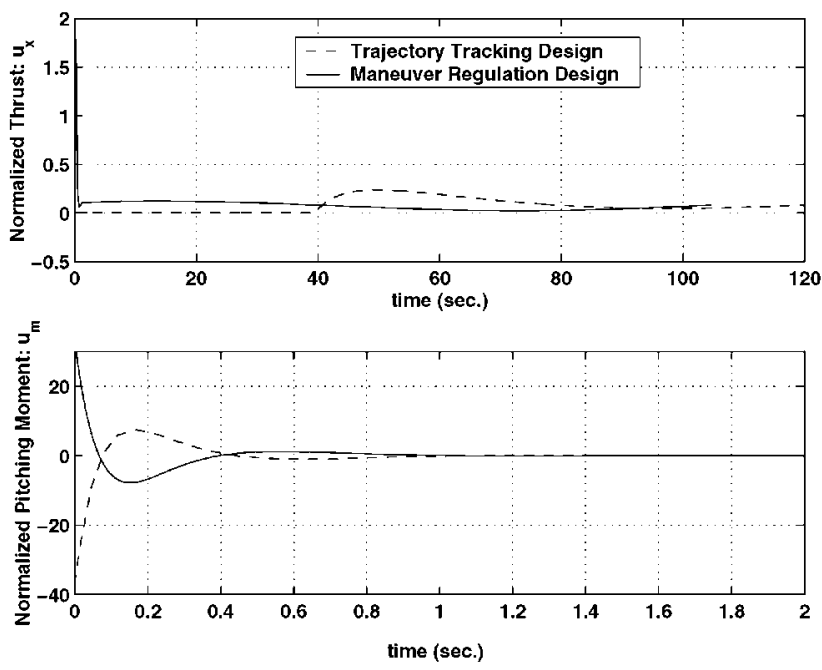

Fig. 5. Thrust and pitching moment required by path tracking and that required by trajectory tracking control for NOE maneuver for the longitudinal aircraft model, non-zero initial errors 


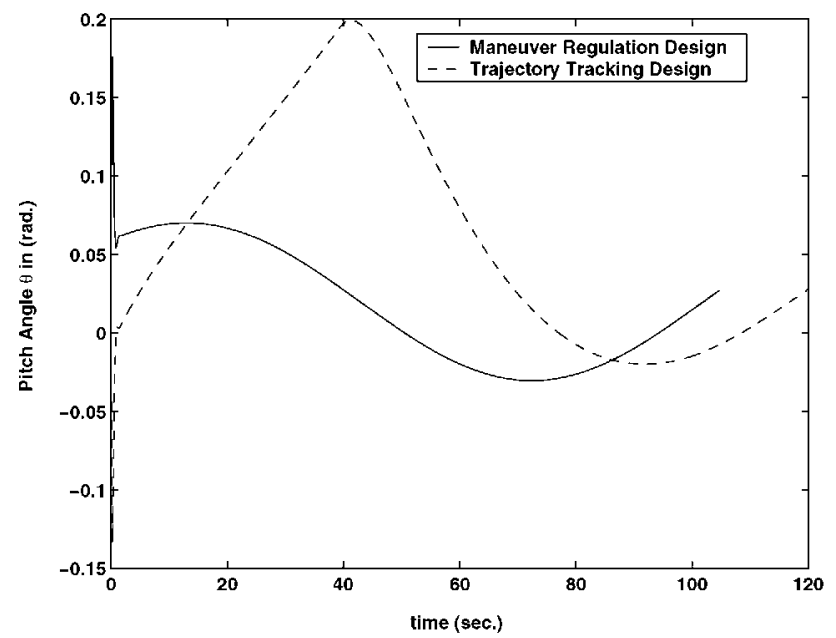

Fig. 6. Longitudinal pitch angle responses for NOE maneuver using path tracking and trajectory tracking controllers, non-zero initial errors

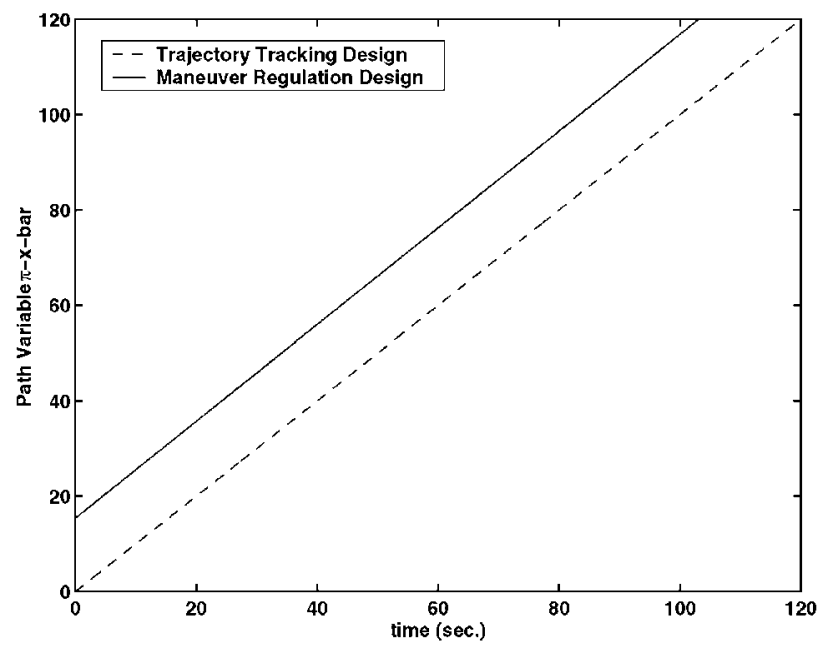

Fig. 7. Path variable $\pi(\bar{x})$ responses for NOE maneuver for the longitudinal aircraft, non-zero initial errors

$8,0,0.0280,0.0027)$. Figure 4 shows the desired NOE path, the path that results from trajectory tracking design and the path that results form the path tracking design. Note that the trajectory tracking controller induces large deviations from the desired path because of the large initial errors. In fact the aircraft stalls in this case. This is obvious from Fig. 5 where the thrust is zero for almost 40 seconds and this cause the aircraft speed to drop below $50 \mathrm{~m} / \mathrm{s}$; the lower part of Fig. 5 shows the initial transient pitching moment only over $2 \mathrm{sec}$. On the other hand, we see in Fig. 4 that the path tracking design performs perfectly and tracks the desired path with negligible deviation. The reason for this is that the aircraft initial conditions correspond to a point on the desired path. Thus the path tracking controller detects that the aircraft is on the desired path and hence control the aircraft to remain on the desired path starting from that point. Figure 6 shows that the response of the pitch angle (driven dynamics) is bounded. Figure 7

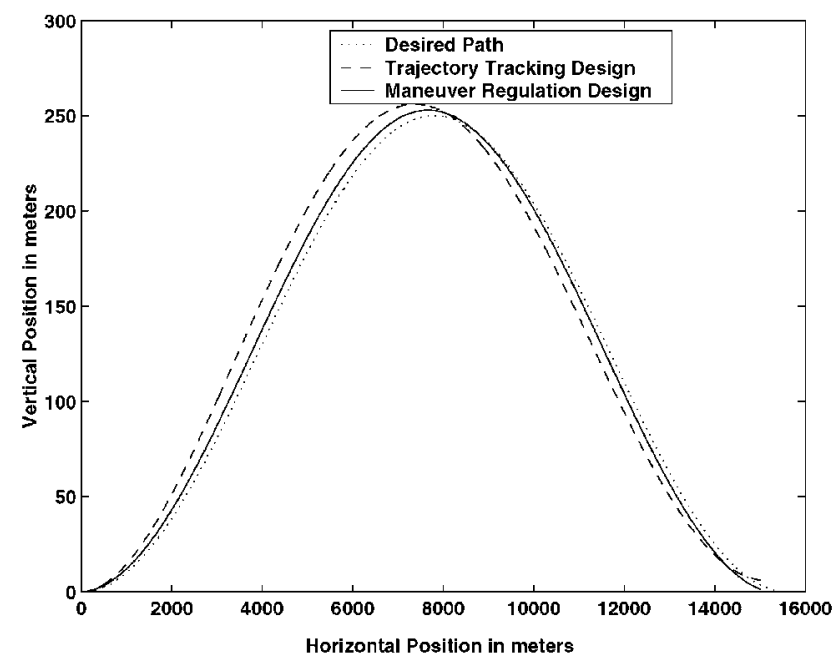

Fig. 8. Trajectory tracking responses and path tracking responses for NOE maneuver for the longitudinal aircraft model, $20 \mathrm{~m} / \mathrm{s}$ wind disturbance

shows the behavior of the path variable $\pi(\bar{x})$ described by equation (33). Because the aircraft initial conditions correspond to a point on the desired path, the response of the path variable is $\pi(\bar{x})=t+c$, where $c$ is a constant.

Next we study the effect of a horizontal wind disturbance of magnitude $20 \mathrm{~m} / \mathrm{s}$ on the closed loop performance of the trajectory tracking design and the path tracking design. Simulations in this case are obtained using zero initial errors namely $\bar{x}(0)=(0,130,0,0,0.0280,0.0027)$. As shown in Fig. 8, the closed loop performance of the trajectory tracking design is significantly degraded by the wind disturbance of $20 \mathrm{~m} / \mathrm{s}$. On the other hand the path tracking design compensate for the wind disturbance and perfectly maintains the aircraft on the desired path. This advantage of path tracking control can be explained by studying the response of the path variable $\pi(\bar{x})$ described by equation (33). Note that in the absence of wind disturbance and initial errors, the trajectory tracking design and the path tracking design produce the same result. In other words the rate of change of the path variable $\dot{\pi}(\bar{x})$ $=1$ or $\pi(\bar{x})=t$. Figure 11 shows the behavior the rate of change of the path variable $\dot{\pi}(\bar{x})$ under the influence of the wind disturbance. Because the wind is in the opposite direction of the desired aircraft motion, the rate of change of the path variable $\dot{\pi}(\bar{x})$ is less than 1, indicating that the commands are generated at a rate slower than that the nominal rate $\dot{\pi}(\bar{x})=1$.

In Fig. 9 we show the control signals required to execute the desired maneuver under the influence of the wind disturbance. Figure 10 shows that the response of the pitch angle (driven dynamics) is bounded.

\section{CONCLUSIONS}

In this paper we have studied a flight control problem for a nonlinear non-minimum phase longitudinal aircraft 

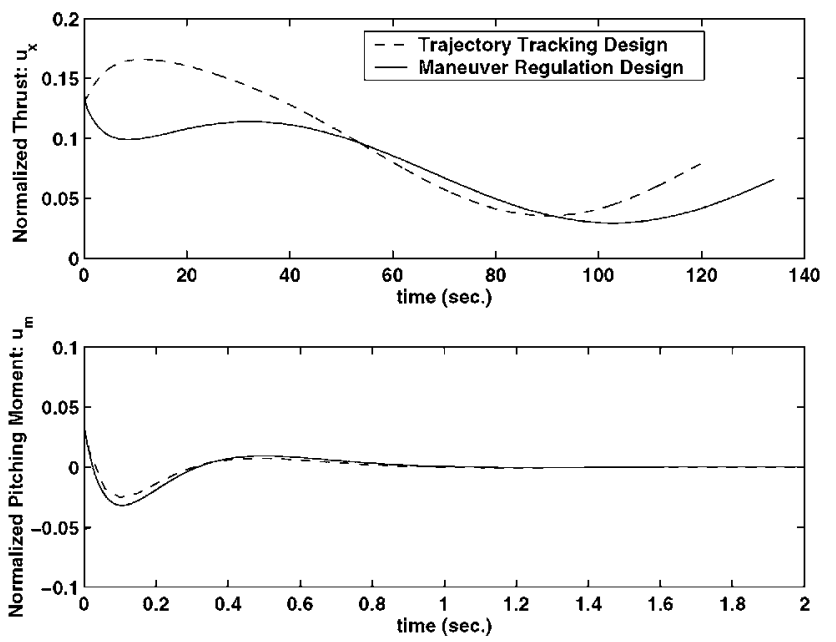

Fig. 9. Thrust and pitching moment required to execute NOE maneuver using path tracking and trajectory tracking controller for the longitudinal aircraft model, $20 \mathrm{~m} / \mathrm{s}$ wind disturbance

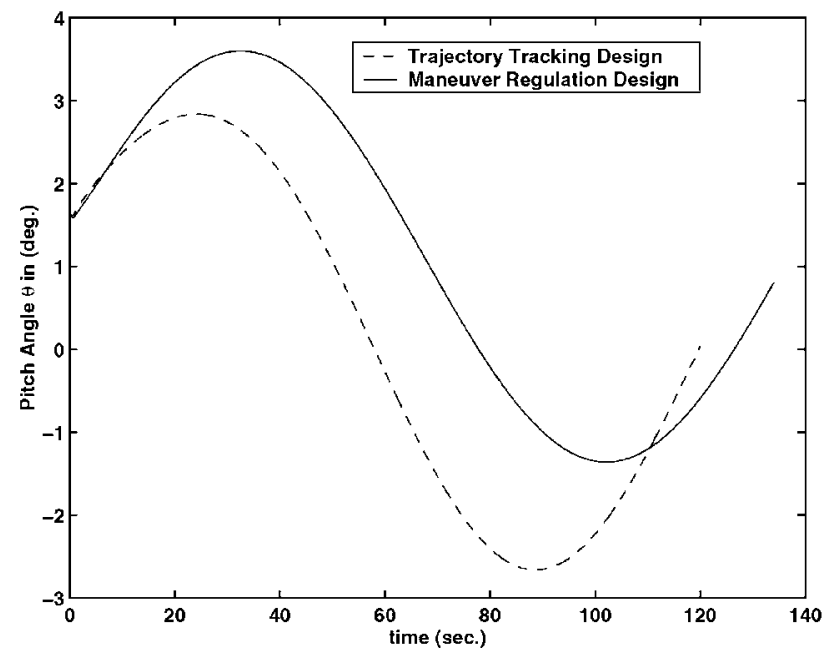

Fig. 10. Longitudinal aircraft pitch angle responses for NOE maneuver using path tracking and trajectory tracking controller, $20 \mathrm{~m} / \mathrm{s}$ wind disturbance

model. In particular, we have studied the execution of a maneuver for which the aircraft is intended to follow a path in a vertical plane corresponding to a specified change in altitude. First we formulate the problem as a nonlinear output trajectory tracking control problem. Next we formulate a related path tracking problem. A controller is developed for this case, and it is compared with a controller that solves the related output trajectory tracking problem. The advantages of the path tracking controller are found to be (a) smaller control values are typically required thereby avoiding control saturation effects and (b) improved robustness is obtained, e.g. as determined by the disturbance rejection. The simulation examples that have been provided clearly confirm these conclusions.

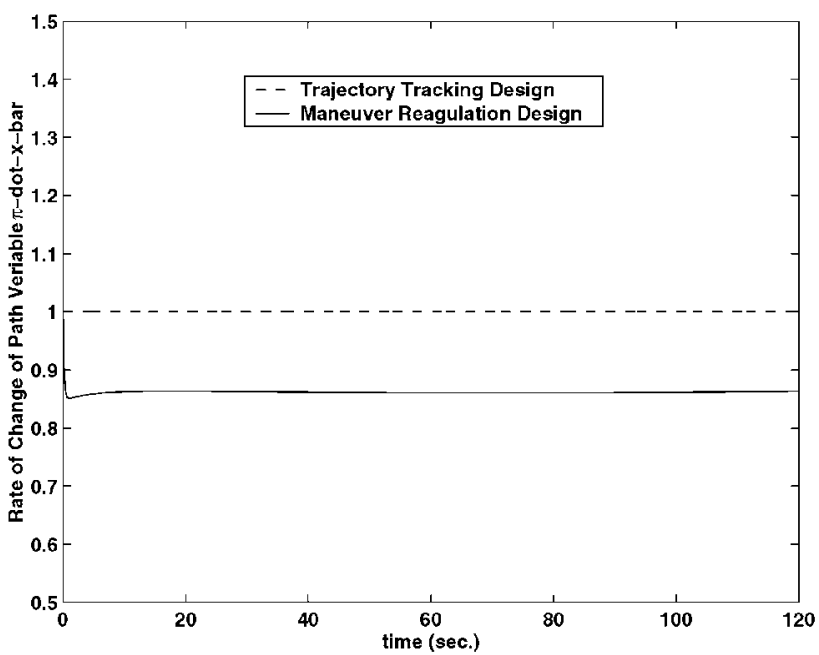

Fig. 11. Rate of change of path variable $\dot{\pi}(\bar{x})$ responses for NOE maneuver for the longitudinal aircraft model, $20 \mathrm{~m} / \mathrm{s}$ wind disturbance.

\section{REFERENCES}

1. Saif A. Al-Hiddabi, "Position Tracking and Path Following for Flight Vehicles Using Nonlinear Control," Ph.D. Thesis, The University of Michigan (2000).

2. Saif A. Al-Hiddabi and N.H. McClamroch, "A Decomposition Approach to Output Tracking for Multivariable Nonlinear Non-minimum Phase Systems," Proc. Amer. Contr. Conf., Philadelphia, PA, pp. 11281132 (1998).

3. — - "Trajectory Tracking Control and Maneuver Regulation Control for the CTOL Aircraft Model," Proc. 38th IEEE Conf. Decis. Contr., Phoenix, Arizona, pp. 1958-1963 (1999).

4. —_, "Aggressive Longitudinal Aircraft Trajectory Tracking Using Nonlinear Control," Submitted to $J$. Guid. Contr. Dyn (2000).

5. Saif A. Hl-Hiddabi, Jinglai Shen, and N.H. McClamroch, "A Study of Flight Maneuvers for the PVTOL Aircraft Model," Proc. Amer. Contr. Conf., San Diego, CA, Vol. 4, pp. 2727-2731 (1999).

6. —_ "Tracking and Maneuver Regulation Control for Nonlinear Non-minimum Phase Systems," Proc. Amer. Contr. Conf., Chicago, Illinois, pp. 492-496 (2000).

7. DeSantis, R.M., "Modeling and Path-tracking for a Load-haul-dump Mining Vehicle," J. Dyn. Syst. Meas. Contr., Vol. 199, pp. 40-47 (1997).

8. Devasia, S., D. Chen, and B. Paden, "Nonlinear Inversion-based Output Tracking," IEEE Trans. Automat. Contr., Vol. 41, No. 7, pp. 930-942 (1996).

9. Hauser, J. and R. Hindman, "Maneuver Regulation from Trajectory Tracking: Feedback Linearizable Systems," Proc. IFAC Symp. Nonlin. Contr. Syst. Design, Nolcos, Tahoe City, CA, pp. 638-643 (1995). 
10. —_, “Aggressive Flight Maneuvers," Proc. IEEE Conf. Decis. Contr., San Diego, CA, pp. 4186-4191 (1997).

11. _ _ "Stability of Maneuver Regulation Using Arbitary Transverse Foliations," Proc. Amer. Contr. Conf., San Diego, CA, pp. 4558-4562 (1999).

12. Kaminer, I., A. Pascoal, E. Hallberg and C. Silvestre, "Trajectory Tracking for Autonomous Vehicles: An Integrated Approach to Guidance and Control," J. Guid. Contr. Dyn., Vol. 21, No. 1, pp. 29-38 (1998).

13. McClamroch, N.H. and Saif Al-Hiddabi, "A Decomposition Based Control Design Approach to Output Tracking for Multivariable Nonlinear Non-minimum Phase Systems," Proc. IEEE Conf. Contr. Appl., Trieste, Italy (1998).

14. D. Brett Ridgely and Michael B. McFarland, "Tailoring Theory to Practice in Tactical Missile Control,' IEEE Contr. Syst. Mag., 19, No. 6, pp. 49-55 (1999).

15. Tran, T., N. Xi and A.K. Bejczy, "Path-based Approach to Integrated Planning and Control for Robotic Systems," Automatica, Vol. 32, No. 12, pp. 16751687 (1996).



Saif A. Al-Hiddabi received his B. Eng. degree in Mechanical Engineering from the Sultan Qaboos University, Sultanate of Oman in 1992, the M.Sc. degree in Aerospace Engineering from the University of Michigan, Ann Arbor, USA, in 1995, M.Sc. degree in Electrical Engineering (Systems) from the University of Michigan, Ann Arbor, USA, in 2000 and Ph.D degree in Aerospace
Engineering from the University of Michigan, Ann Arbor, USA, in 2000.

He is currently assistant professor in the Department of Mechanical and Industrial Engineering at the Sultan Qaboos University of Oman. His research interest include dynamics and control of nonlinear systems with application to mechanical systems and flying vehicles.



N. Harris McClamroch received a Ph.D. degree in engineering mechanics, from The University of Texas at Austin. Since 1967 he has been at The University of Michigan, Ann Arbor, Michigan, where he is a Professor and past Chair of the Department of Aerospace Engineering. He has published numerous papers in the areas of robustness, optimal control, digital feedback, statistical estimation, stochastic control, and nonlinear control. He has worked on many control engineering problems arising in flexible space structures, robotics, automated manufacturing, control technologies for buildings and bridges, and aerospace flight systems. Dr. McClamroch is a Fellow of the IEEE, he received the Control Systems Society Distinguished Member Award, and he is a recipient of the IEEE Third Millennium Medal. He has served as Associate Editor and Editor of the IEEE Transactions on Automatic Control, as Program Chair for the 1994 Conference on Decision and Control, and as General Chair of the 1999 Conference on Control Applications. He has held numerous positions in the IEEE Control Systems Society, including Vice President for Publication Activities, Vice President for Financial Activities, and President. 